\title{
Two-Phase Flow in the Natural Circulation of a Pressurized Water Reactor
}

\author{
Mohammad W. Abdulrahman', Mikdam M. Saleh² \\ ${ }^{1}$ Rochester Institute of Technology \\ Dubai, UAE \\ mwacad@rit.edu; mikdam2@yahoo.com \\ ${ }^{2}$ University of Baghdad \\ Baghdad, Iraq
}

\begin{abstract}
In this paper, natural circulation phenomena in a pressurized water reactor during a small break loss of coolant accident is investigated analytically. By using a one-dimensional analyses and quasi-steady state for two-phase flow, the values of the mass flow rate versus the primary coolant pressure for different core power are determined. It is found that the mass flow rate is reduced suddenly when the two-phase natural circulation is started. Also, it is found that the effect of the core power on mass flow rate is insignificant in the two-phase natural circulation. The analytical results of this paper are compared with previous experimental results and show a very good agreement.
\end{abstract}

Keywords: Natural circulation; PWR; Two-phase; LOCA

\section{Introduction}

It is strongly believed that the natural circulation in a PWR type system can provide an effective mechanism for the rejection of core decay heat to the secondary side of the steam generator. The physics of circulation process is a manometric balance between the hydrostatic head in the down-comer driving the core inlet flow, and the pressure losses incurred in venting the resulting two-phase flow from the core outlet to the heat exchanger $[1,2]$.

In the literature, there are different studies in natural circulation. Gruszczynski and Viskanta studied the flow and heat transfer for water in a rectangular loop in which tube bundles served as the source of heat in one vertical leg and as the heat sink in the other vertical leg. The purpose of the work was to gain improved understanding of the behaviour of a natural circulation loop containing tube bundles. Transients and steady-state tests were conducted and an unsteady one-dimensional formulation was used to model the flow. Detailed comparisons were made between the predicted and measured temperatures and good agreement was obtained under transient and steady-state conditions [3].

Steimke carried out an experimental and theoretical investigation of natural circulation of water in a loop where the flow channels are the annuli between three concentric cylindrical surfaces. An analysis was made based on a one-dimensional model. Results were obtained from the numerical solution to the conservation equation of mass, momentum and energy for steady laminar flow. Agreement between the calculated and measured temperatures, velocities, and Nusselt numbers was reasonably good [4]. Britt and Wood carried out experiments with several systems to determine the operational characteristics of a multiple-loop container used to cool nuclear reactor assemblies at the Savannah River plant [5]. Cheng and Rovang studied the steady-state operation and heat transfer characteristics of a closed loop, two-phase thermosyphon system for low grade heat recovery from liquid heat sources [6]. Burchill and Abramson [7], and, Duffey and Sursock [8] identified three distinct modes of natural circulation relevant to small breaks and transients for PWRs as a function of quality: 1) singlephase, 2) two-phase, and 3) reflux condensation. Abdulrahman has investigated the analytical solution of the energy equation in two dimensional steady state condition. He has determined the temperature distributions of the packed bed reactor in terms of the radial and axial dimensions, as well as the temperature distribution as a function of time. $[9,10]$.

The present work is an analytical investigation of a two-phase natural circulation system relevant to a PWR during a small break loss of coolant accident. The model of this paper utilizes the one-dimensional analyses and the quasi-steady state and solves analytically the conservation equations of mass, momentum and energy. In this paper, an expression for the two- 
phase mass flow rate as a function of a primary pressure is developed, as well as the effect of core power on the mas flow rate is investigated.

\section{Steady State One-Dimensional Natural Circulation Flow}

For a flow in a one-dimensional positive $s$-direction, the steady state equations of continuity, momentum, and energy are respectively [11];

$$
\begin{gathered}
\frac{d u}{d s}=-\frac{u}{\rho} \frac{d \rho}{d s} \\
\rho u \frac{d u}{d s}=-\frac{d P}{d s}-F_{s}-\rho g \cos (\theta) \\
\rho A u \frac{d h}{d s}=-\frac{d q}{d s}
\end{gathered}
$$

where $u$ is the flow velocity, $\rho$ is the fluid density, $P$ is the primary pressure, $g$ is the gravitational acceleration, $A$ is the cross sectional area of the flow channel, $h$ is the specific enthalpy, and $q$ is the heat added to (or rejected from) the coolant. In terms of the mass flow rate $W$, the flow velocity $u$ is:

$$
u=\frac{W}{\rho A}
$$

For turbulent flow in a circular tube of diameter $D$, the viscous force per unit volume $F_{S}$ is;

$$
F_{S}=\frac{4 f\left(\frac{1}{2} \rho u^{2}\right)}{D}
$$

where the friction factor $f$ is given by [12]; $f=\frac{0.046}{(R e)^{0.2}}$. Substituting Eqs. (1), (4) and (5) into Eq. (2) and integrate around the loop, the momentum Eq. will be;

$$
\oint \frac{W^{2}}{A^{2}(s)} \frac{d v}{d s} d s=-\oint \frac{d P}{d s} d s-\oint \frac{4\left(\frac{1}{2} f W^{2}\right)}{\rho A^{2}(s) D(s)} d s-\oint \rho g \cos (\theta) d s
$$

where $v$ is the fluid specific volume and $P$ is the primary pressure. The left hand side and the pressure term of Eq. (6) will vanish. For strongly turbulent flow, it can be assumed that the friction coefficient is constant for a given primary pressure. Noting that $A$ and $D$ are given by their average values over the loop and that $d z=d s \cos (\theta)$, then Eq. (6) becomes;

$$
-g \oint \rho d z=\frac{\frac{1}{2}(4 \bar{f}) W^{2}}{\bar{A}^{2} \bar{D}} \oint \frac{d s}{\rho}
$$




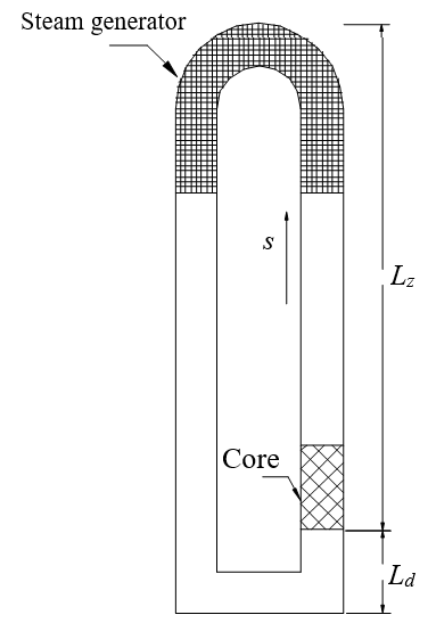

Fig. 1: One-dimensional loop.

From Fig. 1;

$$
\oint \rho d z=\int_{0}^{L_{z}} \rho_{1} d z+\int_{L_{z}}^{0} \rho_{1} d z+\int_{0}^{-L_{d}} \rho_{2} d z+\int_{-L_{d}}^{0} \rho_{2} d z=\int_{0}^{L_{z}} \rho_{1} d z-\int_{0}^{L_{z}} \rho_{2} d z=\left(\bar{\rho}_{1}-\bar{\rho}_{2}\right) L_{z}
$$

and;

$$
\oint \frac{d s}{\rho}=\oint v d s=\bar{v} L_{t}
$$

where $\rho_{1}$ and $\rho_{2}$ are the fluid densities of the system up-flow and down-flow sides respectively, $\bar{\rho}_{1}$ and $\bar{\rho}_{2}$ are the average values of the fluid densities $\rho_{1}$ and $\rho_{2}$ respectively, $\bar{v}$ is the average value of the fluid specific volume, $L_{z}$, and $L_{d}$ are the heights of the PWR and downcomer systems respectively, and $L_{t}$ is the total circulation length. Substituting Eqs. (8) and (9) into Eq. (7) and rearranging, to get;

$$
\frac{\frac{1}{2}(4 \bar{f}) W^{2}}{\bar{A}^{2} \bar{D}} \bar{v} L_{t}=g\left(\bar{\rho}_{2}-\bar{\rho}_{1}\right) L_{z}
$$

Define:

$$
\widehat{W}^{2}=\frac{2 g L_{z} \bar{D} \bar{\rho}_{o}^{2} \bar{A}^{2}}{4 \bar{f}_{o} L_{t}}
$$

where $\bar{D}$ and $\bar{A}$ are the average diameter and cross sectional area of the flow channel respectively. Note that $\widehat{W}$ has the dimensions of flow rate and $\bar{f}_{o}$ and $\bar{\rho}_{o}$ are respectively the overall system average friction coefficient and density corresponding to the conditions at the beginning of NC. Substituting Eq. (11) into Eq. (10) and solving for the mass flow rate, to get; 


$$
W=\frac{\widehat{W}}{\bar{\rho}_{o}} \sqrt{\frac{\left(\bar{\rho}_{2}-\bar{\rho}_{1)}\right.}{\bar{v}} \frac{\bar{f}_{o}}{\bar{f}}}
$$

where $\bar{f}$ is the average friction factor. The value of $\bar{\rho}_{1}$ is obtained from volume averaging of densities over the up-flow side of the PWR system elements, as follows:

$$
\bar{\rho}_{1}=\frac{\bar{\rho}_{c} V_{c}+\bar{\rho}_{u} V_{c}+\bar{\rho}_{s} \frac{V_{s}}{2}}{V_{1}}
$$

where $\bar{\rho}_{c}, \bar{\rho}_{u}, \bar{\rho}_{s}$ are the average densities of the core, sum of the hot leg and upper plenum, and steam generator respectively. $V_{c}, V_{u}, V_{s}$, and $V_{1}$ are the volumes of the core, sum of the hot leg and upper plenum, and steam generator respectively and $V_{1}=V_{c}+V_{u}+\frac{V_{s}}{2}$. The energy Eq. (10) can be written separately for each component of the loop. The term $\left(-\frac{d q}{d s}\right)$ in Eq. (10) is equal to $\left(-\bar{U} \pi D_{s} n\left(T-T_{s e c}\right)\right)$ for the heat sink and zero for the (insulated) pipes. For the heated section, it depends upon the input power distribution. It is well known that the power distribution in a typical PWR core is far from being uniform. To first approximation, the distribution may be taken as sinusoidal. In this paper, to simplify derivations, uniform input power distribution is taken into consideration. For a uniformly distributed input power, the term $\left(-\frac{d q}{d s}\right)$ is equal to $\frac{Q_{o}}{L_{c}}$ for the heated section. Hence, Eq. (10) can be written as:

$$
W \frac{d h}{d s}=\left\{\begin{array}{cc}
Q_{o} / L_{c} & \text { (heat source) } \\
-\bar{U} \pi D_{s} n\left(T_{s}-T_{s e c}\right) & \text { (heat sink) } \\
0 & \text { (insulated pipes) }
\end{array}\right.
$$

where $Q_{o}$ is the heat input power, $L_{c}$ is the length of the core, $\bar{U}$ is the average value of the overall heat transfer coefficient, $D_{s}$ is the steam generator diameter, $n$ is the number of primary tubes of steam generator, $T_{s}$ and $T_{s e c}$ are the steam generator temperatures of the primary and secondary sides respectively.

\subsection{Two-Phase Loop Flow}

In natural circulation, the two-phase mode is regarded to begin when the bubbly flow is carried over into the down-flow side of steam generator and exit piping. Consider the loop to contain only two-phase flow, with unequal densities $\bar{\rho}_{1}$ and $\bar{\rho}_{2}$, on the hot and cold sides, respectively (Fig. 2). 


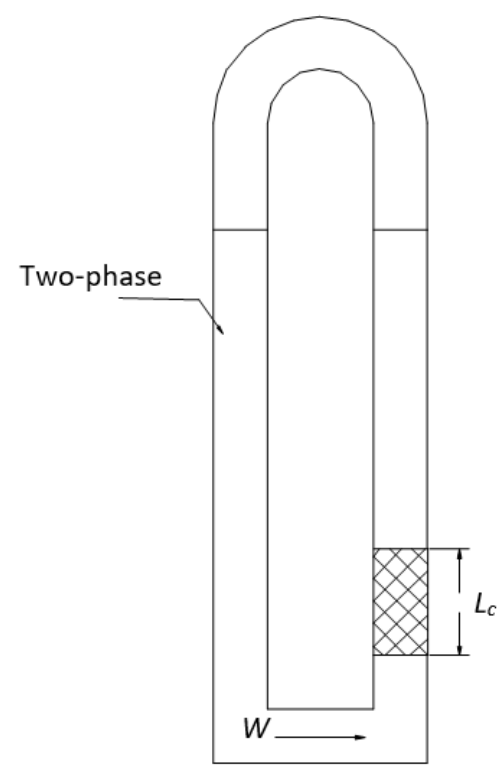

Fig. 2: Two-phase loop.

The solution of Eq. (14c) yields uniform enthalpy and hence quality in the hot leg pipe, which implies, that the outlet core and inlet steam generator qualities are at $x_{o}$. Utilizing the definition of $h=h_{f}+x h_{f g}$ in Eq. (14a), integrating, and using the boundary conditions $\left(x=x_{i}\right.$ at $z=0$ and $x=x_{o}$ at $\left.z=L_{c}\right)$ the quality at the core outlet can be found as;

$$
x_{o}=x_{i}+\frac{Q_{o}}{W h_{f g}}
$$

where $x_{i}$ is the quality at the core inlet, and $h_{f g}$ is the latent heat of vaporization. By solving Eq. (14b) at $z=\frac{L_{s}}{2}$, the quality at the core inlet (steam generator outlet) can be found as;

$$
x_{i}=x_{o}-\frac{\bar{U} \pi D_{s} n\left(T_{s a t}-T_{s e c}\right)}{W h_{f g}} \frac{L_{s}}{2}
$$

Equations (15) and (16) are used now to determine the average density for each elemnet of a PWR loop.

$$
\begin{gathered}
\bar{\rho}_{c}=\frac{W h_{f g}}{v_{f g} Q_{o}} \ln \left[\frac{v_{f}+v_{f g} x_{o}}{v_{f}+v_{f g} x_{i}}\right] \\
\bar{\rho}_{u}=\frac{1}{v_{f}+v_{f g} x_{o}} \\
\bar{\rho}_{s}=\frac{2 W h_{f g}}{v_{f g} \bar{U}_{2} \pi D_{s} n\left(T_{s a t}-T_{s e c}\right) L_{s}} \ln \left[\frac{v_{f}+v_{f g} x_{o}}{v_{f}+v_{f g} x_{o}-\frac{v_{f g} \bar{U}_{2} \pi D_{s} n\left(T_{s a t}-T_{s e c}\right)}{W h_{f g}} \frac{L_{s}}{2}}\right] \\
\bar{\rho}_{2}=\frac{1}{v_{f}+v_{f g} x_{i}}
\end{gathered}
$$


where $\bar{\rho}_{c}, \bar{\rho}_{u}, \bar{\rho}_{s}, \bar{\rho}_{2}$ are the average densities of the core, sum of the hot leg and upper plenum, steam generator, down-flow side respectively, $v_{f}$ is the saturated liquid specific volume, and $v_{f g}=v_{g}-v_{f}$. The average specific volume, $\bar{v}$, in Eq. (12), can be obtained from volume averaging over the PWR system elements, thus;

$$
\bar{v}=\frac{\bar{v}_{c} V_{c}+\bar{v}_{u} V_{u}+\bar{v}_{s} \frac{V_{s}}{2}+\bar{v}_{2} V_{2}}{V_{1}+V_{2}}
$$

where $\bar{v}_{c}, \bar{v}_{u}, \bar{v}_{s}$, and $\bar{v}_{2}$ are the average specific volumes of the core, sum of the hot leg and upper plenum, steam generator, and down-flow side respectively. $V_{c}, V_{u}$, and $V_{s}$, are the volumes of the core, sum of the hot leg and upper plenum, and steam generator respectively, $V_{1}=V_{c}+V_{u}+\frac{V_{s}}{2}$, and $V_{2}=\frac{V_{s}}{2}+V_{c l}$. The magnitudes of $\bar{v}_{c}, \bar{v}_{u}, \bar{v}_{s}$, and $\bar{v}_{2}$ can be determined from:

$$
\begin{gathered}
\bar{v}_{c}=v_{f}+v_{f g} x_{i}+\frac{v_{f g} Q_{o}}{2 W h_{f g}} \\
\bar{v}_{u}=v_{f}+v_{f g} x_{o} \\
\bar{v}_{s}=v_{f}+v_{f g}\left[\begin{array}{c}
x_{o}-\frac{U_{2} \pi D_{s} n\left(T_{s a t}-T_{s e c}\right)}{2 W h_{f g}} \frac{L_{s}}{2} \\
\bar{v}_{2}=v_{f}+v_{f g} x_{i}
\end{array}\right.
\end{gathered}
$$

By solving equations (15) and (16), the following expression can be obtained:

$$
Q_{o}=\bar{U} \pi D_{s} n\left(T_{s a t}-T_{s e c}\right) \frac{L_{s}}{2}
$$

Since, in nucleate boiling, $\bar{U}$ is a function of a primary pressure, $P$ and the other parameters in equation (26) are constants, so that, it can be concluded that, for two-phase flow, the primary pressure, $P$, is constant and can be evaluated as the same value of the primary pressure at the peak mass flow rate. At this stage, one proceeds to evaluate the average densities in the hot and cold legs, $\bar{\rho}_{1}$ and $\bar{\rho}_{2}$, and the average specific volume, $\bar{v}$. The mass flow rate, $W$, can be obtained from equation (12). It should be kept in mind that the temperature difference across the core will be zero, since the inlet and outlet temperatures are at saturation temperature.

\subsection{Overall Heat Transfer Coefficient}

The overall heat transfer coefficient for the radial flow of heat across a circular tube based on the internal tube surface, $U_{i}$, is [13];

$$
\frac{1}{U_{i}}=\frac{1}{H_{i}}+\frac{A_{i} t_{w}}{A_{m w} K_{w}}+\frac{A_{i}}{A_{o} H_{o}}
$$

where $H_{i}$ and $H_{o}$ are the heat transfer coefficient inside and outside the tube respectively, $A_{i}$ and $A_{o}$ are the internal and external tube surface area respectively, $A_{m w}$ is the mean wall area for heat transfer, and $K_{w}$ is the thermal conductivity of the wall tube. The heat transfer resistance by conduction can be neglected because of small thickness of steam generator tubes. For two phase flow in the steam generator, convective heat transfer on the inside of the tubes and nucleate boiling on the secondary side of the tubes will occur [14]. The heat transfer coefficients in the primary side of steam generator $H_{i}$, have been taken, from forced flow correlations. From the above considerations, the average overall heat transfer coefficient for two-phase flow, $\bar{U}$, can be written as; 


$$
\bar{U}=\frac{1}{H_{i 2 \phi}}+\frac{A_{i}}{A_{o} H_{o 2 \phi}}
$$

where; $H_{i 2 \phi}$ is the heat transfer coefficient for two-phase in the primary side of steam generator for bubble flow, which can be taken from the following correlation [2]:

$$
H_{i 2 \phi}=2.5551 \times 10^{-3} e^{0.6446133 P}\left(T_{s a t}-T_{s e c}\right)^{3}
$$

$H_{o 2 \phi}$ is the heat transfer coefficient for nucleate boiling of the secondary side of steam generator, which can be taken from the following correlation [2]:

$$
H_{o 2 \phi}=2.5551 \times 10^{-3} e^{0.6446133 P_{s e c}}\left(T_{\text {sat }}-T_{\text {sec }}\right)^{3}
$$

where $P_{\mathrm{sec}}$ is the secondary side pressure in $\mathrm{MPa}, T$ in ${ }^{\circ} \mathrm{C}$ and $H_{\mathrm{o}}$ in $\frac{\mathrm{kW}}{\mathrm{m}^{2} .{ }^{\circ} \mathrm{C}}$. To evaluate the two phase Reynolds number, two phase dynamic viscosity, $\mu_{2 \emptyset}$, must be determined from the following equation:

$$
\frac{1}{\mu_{2 \phi}}=\frac{\bar{x}_{s}}{\mu_{g}}+\frac{1-\bar{x}_{s}}{\mu_{f}}
$$

where; $\bar{x}_{S}$ is the average quality of stream generator which is equal to $\frac{x_{i}+x_{0}}{2}$.

\section{Results}

In this paper, in order to obtain the results of the NC parameters, iterations are used. The values of $\rho_{o}$ and $f_{o}$ for every reactor power $Q_{o}$, can be determined when the primary pressure is at its initial value $\left(P_{o}=11.2 \mathrm{MPa}\right)$ (see Table 1$)$.

Table 1: Values of $\bar{\rho}_{o}$ and $\bar{f}_{o}$ as a function of core power.

\begin{tabular}{|c|c|c|c|}
\hline $\boldsymbol{Q}_{\boldsymbol{o}}(\mathbf{K W})$ & 30 & 60 & 100 \\
\hline$\overline{\boldsymbol{\rho}}_{\boldsymbol{o}}$ & 743.2258 & 734.6119 & 730.6987 \\
\hline$\overline{\boldsymbol{f}}_{\boldsymbol{o}}$ & $4.847 \times 10^{-3}$ & $4.614 \times 10^{-3}$ & $4.462 \times 10^{-3}$ \\
\hline
\end{tabular}

The results of this paper are compared with the experimental data from the Semiscale Mod-2A system for three different powers (30, 60 and $100 \mathrm{KW)} \mathrm{[14].} \mathrm{The} \mathrm{data} \mathrm{required} \mathrm{for} \mathrm{the} \mathrm{calculations} \mathrm{in} \mathrm{this} \mathrm{paper} \mathrm{that} \mathrm{concern} \mathrm{the} \mathrm{Semiscale} \mathrm{Mod-2A}$ system are shown in Table $2[2,8,14]$. Figures 3 and 4 show the calculated mass flow rate of the natural circulation as a function of the primary pressure as well as the comparisons of the calculated results with the experimental results of Semiscale Mod-2A system.

Table 2: Summary of the design and operating data for the Semiscale model.

\begin{tabular}{|c|c|c|c|c|c|}
\hline Parameter & Data & Parameter & Data & Parameter & Data \\
\hline$A_{i}$ & $3.05 \times 10^{-4} \mathrm{~m}^{2}$ & $L_{\mathrm{s}}$ & $20 \mathrm{~m}$ & $P_{\text {sec }}$ & $6 \mathrm{MPa}$ \\
\hline$A_{o}$ & $3.87 \times 10^{-4} \mathrm{~m}^{2}$ & $V_{\mathrm{c}}$ & 0.06 & $T_{\text {sec }}$ & $275.64^{\circ} \mathrm{C}$ \\
\hline$D_{\mathrm{s}}$ & $0.0222 \mathrm{~m}$ & $V_{\mathrm{u}}$ & 0.18 & $T_{1}$ & $T_{\text {bulk }}$ \\
\hline$t_{w}$ & $0.00124 \mathrm{~m}$ & $V_{\mathrm{s}}$ & 0.22 & $\rho_{1}$ & $\rho_{\text {bulk }}$ \\
\hline$n$ & 6 & $V_{\mathrm{cl}}$ & 0.29 & $\widehat{W}$ & $1.35 \mathrm{Kg} / \mathrm{sec}$ \\
\hline$L_{\mathrm{c}}$ & $3.66 \mathrm{~m}$ & full core power (scaled) & $2 \mathrm{MW}$ & & \\
\hline \multicolumn{4}{|r}{} \\
\hline
\end{tabular}




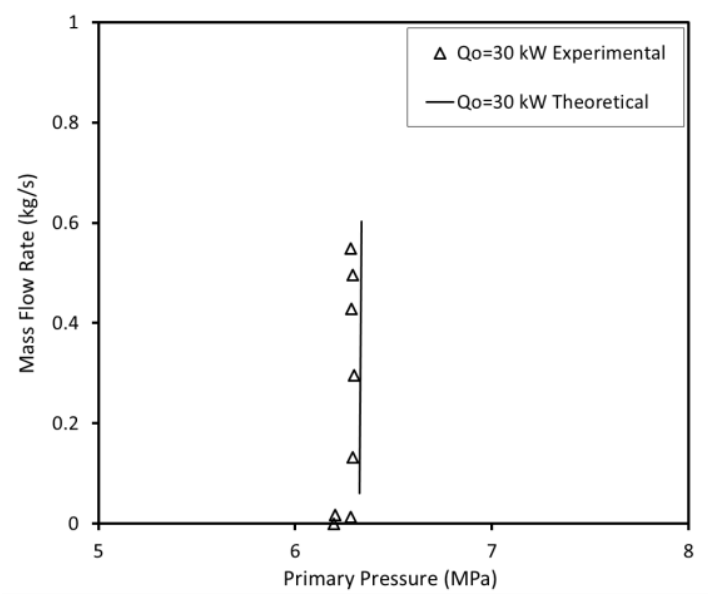

(a)

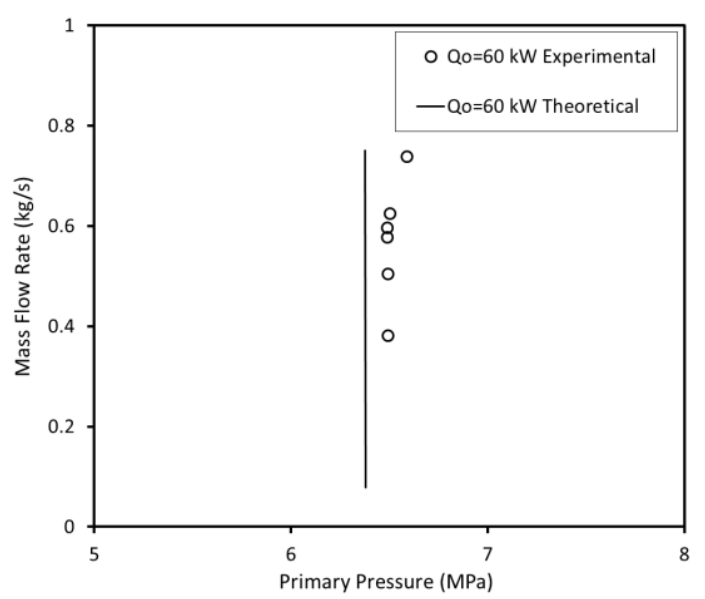

(b)

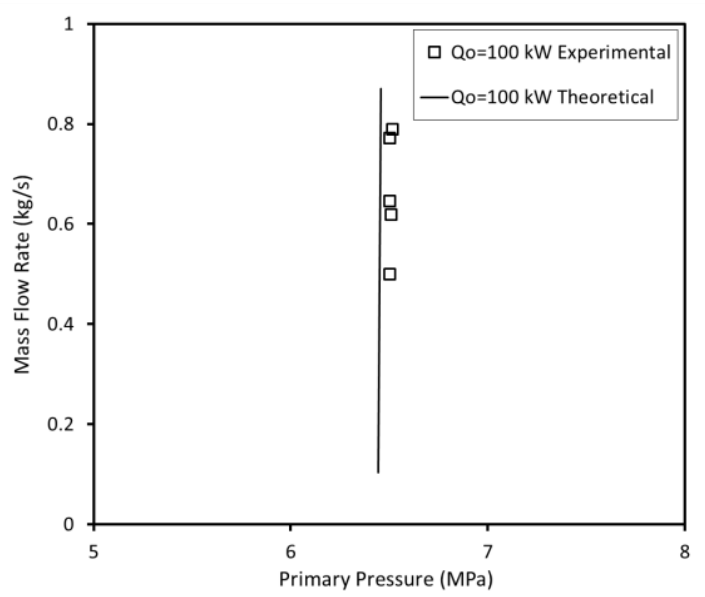

(c)

Fig. 3: Comparison of theory with Semiscale PWR data of Mass flow rate versus Primary pressure for different core powers (a) $Q_{o}=$ $30 \mathrm{~kW}$, (b) $Q_{o}=60 \mathrm{~kW}$, (c) $Q_{o}=100 \mathrm{~kW}$. 


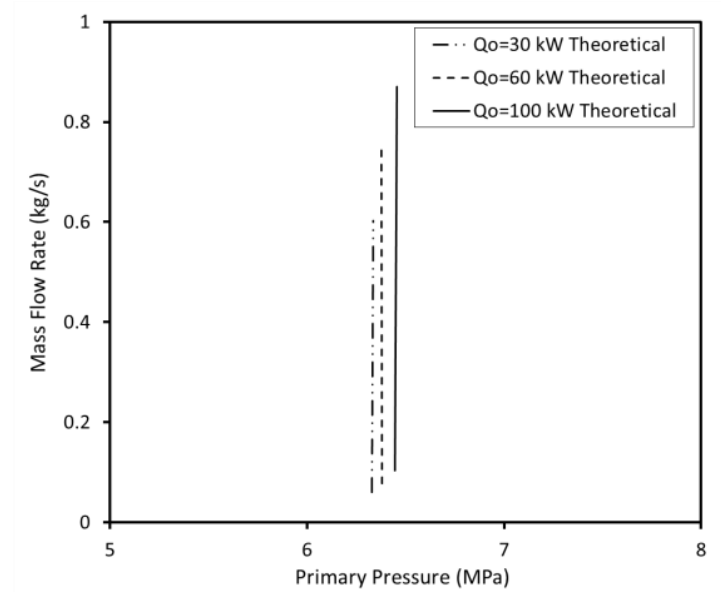

Fig. 4: Effect of core power on Mass flow rate versus Primary pressure.

In PWR, NC starts when all the primary coolant is at single-phase. When the draining in the primary loop is continued, the lever of the coolant will decrease until it reaches the hot leg where combined single and two-phase flow in the NC will start. As more fluid is drained in the primary loop of the PWR, the bubbly flow will eventually be carried over into the steam generator down-flow side and exit piping. In this case, the whole primary coolant will be in a two-phase mode.

In Fig. 3, it can be seen that in two-phase flow, the mass flow rate is sharply reduced as the overall driving head is drastically reduced by the presence of two-phase flow in the down-flow side of the steam generator. The cold leg temperature will remain at the same saturated temperature because the pressure will remain constant for two-phase flow. Since both the inlet and outlet temperatures are at saturation, then the core temperature difference will be reduced to zero. Figure 4 shows the effect of the reactor power on the NC parameters. In general, it can be seen that the overall trends for the three power levels are similar. The mass flow rate at the starting of the two-phase NC, is greater the higher the core power, while at the ending point is approximately the same.

Table 3 shows the maximum error between the experimental and theoretical results of the primary pressure and the NC mass flow rate. The discrepancies in the results can be attributed to different reasons. The first one is the uncertainties in experimental data, which were estimated [8] as $\pm 0.033 \mathrm{Kg} / \mathrm{s}$ for mass flow rate, and $\pm 0.4 \mathrm{MPa}$ for secondary pressure. The second reason is the model approximations. The one dimensional model used is inadequate, under certain operating conditions and in regions where abrupt changes in flow area occurs, to describe the three dimensional nature of the flow. Another reason is the usage of forced flow correlations for heat transfer coefficient and friction factor in NC flow and neglecting the effect of form losses in the various components in the loop.

Table 3: Maximum absolute errors between experimental and theoretical results for the primary pressure versus NC mass flow rate for different power values.

\begin{tabular}{|c|c|}
\cline { 2 - 2 } \multicolumn{1}{c|}{} & Max. error (\%) \\
\hline Power (KW) & $\boldsymbol{P}$ \\
\hline 30 & 2.1 \\
\hline 60 & 3.2 \\
\hline 100 & 0.96 \\
\hline
\end{tabular}

\section{Conclusion}

In this paper, one dimensional continuity, momentum and energy equations are solved to find the mass flow rate of the NC flow versus the primary pressure in a PWR plant. It is found that the mass flow rate of the two-phase NC flow is decreasing sharply with decreasing the primary pressure. Also, it is found that the NC mass flow rate is slightly dependent on core power. The modelling results of this paper are compared with previous experimental data and show a good agreement. 
It can be concluded that the quasi steady-state one dimensional analyses of this paper can be used to describe the two-phase NC phenomena in a PWR during a small break loss of coolant accident.

\section{References}

[1] L. W. Ward, "Simplified small-break blowdown models," Trans. Am. Nucl. Soc., vol. 18, p. 232, 1974.

[2] D. J. Shimeck and G. W. Johnsen, "Natural circulation cooling in a pressurized water reactor geometry aunder accident-induced conditions," Nucl. Sci. Eng., vol. 88, pp. 311-320, 1984.

[3] M. J. Gruszczynski and R. Viskanta, "Heat transfer from a vertical tube bundle under natural circulation conditions," in Proceedings of the ASME-JSME Thermal Engineering Joint conference Processing, Y. Mori, and W. J. Yang, eds., 1983, vol. 3, pp. 403-410.

[4] J. L. Steimke, "Natural convection heat transfer for a concentric tube thermosyphon," ASME Journal of Heat Transfer, vol. 107, pp. 583-588, 1985.

[5] T. E. Britt, and D. C. Wood, "Free convection in a partially submerged fluid loop," ASME paper No. 83-HT-67, 1983.

[6] K. C. Cheng, and G. W. Rovang, "Operating and heat transfer characteristics of a closed-loop to-thermosyphon system for low grade waste heat recovery," in Proceedings of the ASME-JSME Thermal Engineering Joint conference Processing, P. J. Marto and I. Tanasawa, eds., ASME, vol. 3, pp. 179-186.

[7] W. E. Burchill, and P. E. Abramson, PWR small break LOCA. Guidebook to light water reactor safety analysis, Chap. 3, P. B. Abramson, ed., Hemisphere, New York, 1985.

[8] R. B. Duffey and J. P. Sursock, "Natural circulation phenomena to small breaks and transients," Nuclear Engineering and Design, vol. 102, pp. 115-128, 1987.

[9] M. W. Abdulrahman, "Heat transfer in a tubular reforming catalyst bed: Analytical modelling," in Proceedings of the 6th International Conference of Fluid Flow, Heat and Mass Transfer (FFHMT'19), 2019.

[10] M. W. Abdulrahman, "Exact analytical solution for two-dimensional heat transfer equation through a packed bed reactor," in Proceedings of the $7^{\text {th }}$ World Congress on Mechanical, Chemical, and Material Engineering (MCM'20), 2020.

[11] J. B. Keller, “oscillations in a model of thermal convection,”. J. Fluid Mech., Vol. 26, pp. 599-606, 1966.

[12] A. Shitzer, D. Kalmanoviz, Y. Zvirin and G. Grossman, "Experiments with a flat-plate solar water heating system in thermosyphonic flow," Solar Energy, vol. 22, pp. 27-35, 1979.

[13] M. M. El-Wakil, Nuclear power engineering. Mc Graw-Hill Book Company, 1962.

[14] G. G. Loomis and K. Soda, "Results of the Semiscale Mod-2A natural circulation experiments," NRC Report, Idaho National Engineering Lab., NUREG / CR-2335, September, 1982. 